\title{
Nonlinear Robust Control of 3 Phase Inverter with Output LC Filter
}

\author{
Beytullah Okur* Erkan Zergeroglu** Murat Seker ** \\ Enver Taticioglu ${ }^{* * *}$ \\ * Mechatronics Engineering Department, Yildiz Technical University, \\ Istanbul, Turkey (e-mail:okur@yildiz.edu.tr) \\ ** Computer Engineering Department, Gebze Institute of Technology, \\ Kocaeli, Turkey (e-mails: [ezerger, mseker]@bilmuh.gyte.edu.tr) \\ *** Electrical Engineering Department, Izmir Institute of Technology, \\ Izmir, Turkey (e-mail: envertatlicioglu@iyte.edu.tr)
}

\begin{abstract}
Three phase inverters are commonly used to transfer energy from a source to the power grid. The quality of the power delivered to the grid, can be ensured via the use of an output LC filter. However inserting an output filter to an inverter circuitry would introduce new challenges to the controller design due to the additional parametric uncertainties imposed. In this study we present a new model based robust controller for a three phase inverter with output LC filter under the constraint that the output filter parameters are not exactly known. Specifically, $d-q$ reference frame model of an inverter with output LC filter is used to develop a nonlinear robust controller that ensures the 3-phase output voltage with desired amplitude and frequency and with lowest harmonic distortion. Stability of the proposed method and the boundedness of the closed-loop system, is established via Lyapunov based tools in conjunction with a robust backstepping procedure. Simulation results are given in order to demonstrate performance and effectiveness of the proposed robust controller.
\end{abstract}

Keywords: Three phase inverter, output LC filter, model based robust control.

\section{INTRODUCTION}

In recent years, renewable energy sources are getting more attention Lawrence and Middlekauff [2005]. As a consequence, solar cells and wind tribunes are becoming widely applied in distributed generation systems Eltawil and Zhao [2010]. Generally, electrical power produced by these type of energy sources are stored in chemical (i.e. batteries) or electrical (super capacitors) storage devices and connected to the power grid via a $d c$-to- $a c$ inverter system. To connect the inverter output efficiently to the grid, the amplitude and the frequency of the inverter output need to be matched with the grid side Blaabjerg et al. [2006]. An LC low-pass filter is usually connected to the output of the inverter to achieve the lowest Total Harmonic Distortion (THD) Ahmed et al. [2007]. The inverter-filter couple needs to be actively controlled to ensure the lowest THD of output voltage with the desired amplitude and frequency under the different load conditions Kim and Sul [2011].

Power quality is one of the major performance requirement for inverters. Performance of the controller algorithm has direct influence on the power quality (see Prodanovic and Green [2002] and Kovari et al. [2004]). Model-based controllers have been implemented in the literature, to name a few, Kukrer [1996] implement the discrete-time deadbeat control algorithm by using space vector based model of an inverter with output LC filter. In Mattavelli [2005] an improved dead-beat controller with an disturbance observer is proposed and a state-space model of the inverter is used. In Escobar et al. [2007], authors used an adaptive controller based on neural-network identification and deadbeat current regulation. Cortes et al. [2009] proposed a model predictive controller with an observer for the load current estimation. In Kim and Lee [2010], a nonlinear model of an inverter including output LC filter is derived and input-output feedback linearization is applied to the model. In Mu et al. [2011], a nonlinear controller requiring the exact values of the model parameters was proposed. A flatness based control method was proposed in Houari et al. [2012]. Wai et al. [2013] applied a backstepping control design procedure.

In this work, we propose a model based nonlinear robust controller based on the $d-q$ reference frame model of an inverter with output LC filter. Despite the lack of exact knowledge of the parameters of the output filter, the proposed robust controller ensures practical 3-phase output voltage tracking to any desired amplitude and frequency. The stability of the overall system is proven via Lyapunov based tools and effectiveness of the proposed method is illustrated through simulation studies.

The rest of the work is organized in the following manner: $d-q$ reference frame model of the investigated system is given Section 2. Section 3 presents the objective and details of the design procedure while Section 4 contains the stability analysis. Results of our simulation studies are presented in Section 5 and Section 6 contains some concluding results. 


\section{INVERTER MODEL}

An illustration of the system investigated in this work is presented in Figure 1. The system consists of a three legged full bridged intelligent power module (IPM), a three phase output LC filter and a three phase load. For the sake of robustness, the parameters of the load are assumed to be unknown. In Figure $1, V_{d c}$ and $C_{d c}$ are the DC link voltage and capacitance, respectively, $S_{a}, S_{b}, S_{c}$ are the switching functions of the gates of IGBTs in the IPM. The gates of the IGBTs are switched by PWM signals according to the control input signal. $V_{a}, V_{b}, V_{c}$ are the output voltages, $i_{a}, i_{b}, i_{c}$ are the output currents of the switching elements. The output voltages and currents of the LC filter are labelled as $V_{a f}, V_{b f}, V_{c f}$ and $i_{L a}, i_{L b}, i_{L c}$.

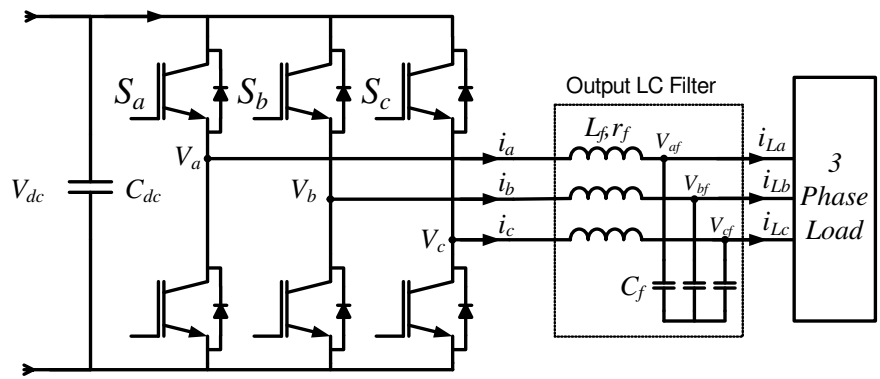

Fig. 1. Inverter with an output LC Filter

As the output of the inverter is periodic, the $d-q$ synchronous reference frame representation of the voltage and the current dynamics of the LC Filter shown in Figure 1 can be written as Houari et al. [2012]

$$
\begin{aligned}
C_{f} \frac{d}{d t} V_{C d} & =C_{f} \omega V_{C q}+i_{d}-i_{L d} \\
C_{f} \frac{d}{d t} V_{C q} & =-C_{f} \omega V_{C d}+i_{q}-i_{L q} \\
L_{f} \frac{d}{d t} i_{d} & =-r_{f} i_{d}+L_{f} \omega i_{q}+V_{d}-V_{C d} \\
L_{f} \frac{d}{d t} i_{q} & =-r_{f} i_{q}-L_{f} \omega i_{d}+V_{q}-V_{C q}
\end{aligned}
$$

where $C_{f} \in \mathbb{R}$ is the filter capacitance, $L_{f} \in \mathbb{R}$ is the filter inductance, $r_{f} \in \mathbb{R}$ is the resistance of the filter inductance, and $\omega(t) \in \mathbb{R}$ is the angular frequency. Note that, $C_{f}, L_{f}, r_{f}$ are considered to be uncertain parameters.

After some mathematical manipulations, the $\mathrm{d}-\mathrm{q}$ frame dynamics in (1)-(4) is rewritten in the following compact form

$$
\begin{aligned}
C_{f} \dot{V}_{C} & =C_{f} W_{s s} V_{C}+I-I_{L} \\
L_{f} \dot{I} & =-r_{f} I+L_{f} W_{s s} I+U-V_{C} .
\end{aligned}
$$

In (5) and (6), $V_{C}(t), I_{L}(t), I(t), U(t) \in \mathbb{R}^{2}$ represent the system output, the load current, the filter input current, and the control input, respectively, defined as

$$
V_{C} \triangleq\left[\begin{array}{c}
V_{C d} \\
V_{C q}
\end{array}\right], I_{L} \triangleq\left[\begin{array}{c}
i_{L d} \\
i_{L q}
\end{array}\right], I \triangleq\left[\begin{array}{c}
i_{d} \\
i_{q}
\end{array}\right], U \triangleq\left[\begin{array}{c}
V_{d} \\
V_{q}
\end{array}\right]
$$

and $W_{s s}(\omega) \in \mathbb{R}^{2 \times 2}$ is an auxiliary skew-symmetric matrix defined as

$$
W_{s s} \triangleq\left[\begin{array}{cc}
0 & \omega \\
-\omega & 0
\end{array}\right]
$$

\section{PROBLEM STATEMENT AND CONTROLLER FORMULATION}

Our aim is to design a controller that ensures the reference input tracking of the output voltage of the inverter with minimum harmonics provided that input current, load current and output voltage of the inverter are measurable, under the restriction that filter parameters are not available. In order to quantify the performance of the controller, we define the output voltage tracking error, $e(t) \in \mathbb{R}^{2}$, as the difference between the reference output voltage, denoted by $V_{\text {Cref }}(t) \in \mathbb{R}^{2}$, with the actual output voltage, as follows

$$
e \triangleq V_{C r e f}-V_{C}
$$

The reference output voltage along with its first and second time derivatives are assumed to be bounded functions of time. Taking the time derivative of (9), multiplying by $C_{f}$, inserting the voltage dynamics presented in (5), and finally adding and subtracting $C_{f} W_{s s} V_{C r e f}$, the following open-loop error dynamics is obtained

$$
C_{f} \dot{e}=C_{f} \dot{V}_{C r e f}+C_{f} W_{s s} e-C_{f} W_{s s} V_{C r e f}-I+I_{L} \text {. }
$$

To insert the dynamics of filter input current $I(t)$, we will apply a backstepping procedure by defining an auxiliary control input like term, denoted by $I_{d}(t) \in \mathbb{R}^{2}$. Adding and subtracting this term to (10) yields

$$
C_{f} \dot{e}=C_{f} \dot{V}_{C r e f}+C_{f} W_{s s} e-C_{f} W_{s s} V_{C r e f}+I_{L}+z-I_{d}
$$

where $z(t) \in \mathbb{R}^{2}$ is an auxiliary error designed to quantify the mismatch between the filter input current $I(t)$ and the auxiliary term $I_{d}(t)$ in the sense that

$$
z \triangleq I_{d}-I \text {. }
$$

Based on the subsequent analysis, the auxiliary control input like term $I_{d}(t)$ is designed as

$$
I_{d}=K_{e} e+\hat{C}_{f}\left(\dot{V}_{C r e f}-W_{s s} V_{C r e f}\right)+I_{L}+V_{R 1}
$$

where $K_{e} \in \mathbb{R}^{2 \times 2}$ is a diagonal, positive definite, constant gain matrix, $\hat{C}_{f} \in \mathbb{R}$ represents the constant best-guess estimate of $C_{f}$, and $V_{R 1}(e) \in \mathbb{R}^{2}$ is a robustifying term designed in the following manner

$$
V_{R 1}=\frac{\rho_{1}^{2}}{\epsilon_{1}} e
$$

where $\epsilon_{1} \in \mathbb{R}$ is a positive constant and $\rho_{1} \in \mathbb{R}$ is a positive bounding constant that satisfies

$$
\rho_{1}>\left\|\widetilde{C}_{f}\left(\dot{V}_{C r e f}-W_{s s} V_{C r e f}\right)\right\|
$$

with $\widetilde{C}_{f} \triangleq C_{f}-\hat{C}_{f} \in \mathbb{R}$ being the constant parameter estimation error. Inserting (13) into the (11), the closedloop dynamics of the voltage subsystem is obtained as

$C_{f} \dot{e}=C_{f} W_{s s} e-K_{e} e+\widetilde{C}_{f}\left(\dot{V}_{C r e f}-W_{s s} V_{C r e f}\right)+z-\frac{\rho_{1}^{2}}{\epsilon_{1}} e$.

The backstepping design procedure applied requires the dynamics of the auxiliary error signal $z(t)$ to be investigated. To obtain the dynamics of $z(t)$, we take the time derivative of (12), multiply it by $L_{f}$, insert the time 
derivative of (13) and the current dynamics given by (6), and then add and subtract $L_{f} W_{s s} I_{d}$ term, to obtain

$$
L_{f} \dot{z}=Y \varphi+L_{f} W_{s s} z+V_{C}-U
$$

where $Y(t)$ is a regressor matrix and $\varphi$ is an unknown constant parameter vector with their multiplication being defined as

$$
\begin{aligned}
Y \varphi= & r_{f} I-\frac{L_{f}}{C_{f}}\left(I-I_{L}\right) \\
& +L_{f}\left\{\left(K_{e}+\frac{\rho_{1}^{2}}{\epsilon_{1}}\right)\left(\dot{V}_{C r e f}-W_{s s} V_{C}\right)\right. \\
& +\hat{C}_{f}\left(\ddot{V}_{C r e f}-\dot{W}_{s s} V_{C r e f}-W_{s s} \dot{V}_{C r e f}\right) \\
& \left.+\dot{I}_{L}-W_{s s} I_{d}\right\} .
\end{aligned}
$$

Notice that, to calculate the regressor matrix $Y(t)$ the time derivative of $I_{L}(t)$ is required. While this may be considered as a drawback of the proposed work, it is remarked that since we design a robust controller numerical derivative of $I_{L}(t)$ can be utilized in the regressor matrix and the subsequently robustifying term [i.e., (20)] will compensate for this mismatch as well.

Based on the subsequent stability analysis, the control input $U(t)$ is designed to have the following form

$$
U=e+K_{z} z+V_{C}+Y \hat{\varphi}+V_{R 2}
$$

where $K_{z} \in \mathbb{R}^{2 \times 2}$ is a constant, diagonal, positive definite gain matrix, $\hat{\varphi}$ is the constant best-guess estimate of the unknown parameter vector $\varphi$, and $V_{R 2}(\cdot) \in \mathbb{R}^{2}$ is a robustifying term designed in the following form

$$
V_{R 2}=\frac{\rho_{2}^{2}}{\epsilon_{2}} z
$$

where $\epsilon_{2} \in \mathbb{R}$ is a positive constant and $\rho_{2}(\|e\|,\|z\|) \in \mathbb{R}$ is a positive bounding function which satisfies

$$
\rho_{2}>\|Y \widetilde{\varphi}\|
$$

with the constant parameter estimation error defined as $\widetilde{\varphi} \triangleq \varphi-\hat{\varphi}$. After substituting (19) and (20) into the openloop error dynamics in (17), the closed-loop dynamics of $z(t)$ is obtained as follows

$$
L_{f} \dot{z}=L_{f} W_{s s} z+Y \widetilde{\varphi}-e-K_{z} z-\frac{\rho_{2}^{2}}{\epsilon_{2}} z .
$$

\section{STABILITY ANALYSIS}

At this stage we are ready to state the following Theorem: Theorem 1. The robust controller of (19) and the auxiliary control input (13) with the robustifying terms (14) and (20) guarantee uniform ultimate boundedness of the inverter output voltage tracking error $e(t)$ in the sense that

$$
\|e(t)\| \leq \sqrt{\frac{a}{b}\|x(0)\|^{2} \exp (-\beta t)+\frac{2 \epsilon}{b \beta}(1-\exp (-\beta t))}
$$

where $x(t) \triangleq\left[e^{T} z^{T}\right]^{T} \in \mathbb{R}^{4}$ is the combined error signal, $a, b, \beta, \epsilon \in \mathbb{R}$ are positive scalars defined explicitly as

$$
\begin{aligned}
& a \triangleq \max \left\{C_{f}, L_{f}\right\} \\
& b \triangleq \min \left\{C_{f}, L_{f}\right\} \\
& \beta \triangleq \frac{2 \min \left\{\lambda_{\min }\left(K_{e}\right), \lambda_{\min }\left(K_{z}\right)\right\}}{\max \left\{C_{f}, L_{f}\right\}} \\
& \epsilon \triangleq \frac{\epsilon_{1}}{4}+\frac{\epsilon_{2}}{4}
\end{aligned}
$$

where $\epsilon_{1}, \epsilon_{2}, K_{e}$ and $K_{z}$ were previously defined, and the notation $\lambda_{\max }(\cdot)$ and $\lambda_{\min }(\cdot)$ are used to denote maximum and minimum eigenvalue of a matrix, respectively.

Proof. To prove the theorem, we define a non-negative Lyapunov function, denoted by $V(e, z) \in \mathbb{R}$, in the following manner

$$
V \triangleq \frac{C_{f}}{2} e^{T} e+\frac{L_{f}}{2} z^{T} z
$$

which can be bounded from below and above as

$$
\frac{1}{2} \min \left\{C_{f}, L_{f}\right\}\|x\|^{2} \leq V \leq \frac{1}{2} \max \left\{C_{f}, L_{f}\right\}\|x\|^{2} .
$$

The time derivative of the above Lyapunov function is obtained as

$$
\dot{V}=C_{f} e^{T} \dot{e}+L_{f} z^{T} \dot{z}
$$

and after substituting the closed-loop dynamics in (16) and (22), simplifying the resulting equation by canceling the common terms, we reach

$$
\begin{aligned}
\dot{V}= & -e^{T} K_{e} e-z^{T} K_{z} z+C_{f} e^{T} W_{s s} e+L_{f} z^{T} W_{s s} z \\
& +e^{T} \widetilde{C}_{f}\left(\dot{V}_{C r e f}-W_{s s} V_{C r e f}\right)-\frac{\rho_{1}^{2}}{\epsilon_{1}}\|e\|^{2} \\
& +z^{T} Y \widetilde{\varphi}-\frac{\rho_{2}^{2}}{\epsilon_{2}}\|z\|^{2} .
\end{aligned}
$$

Following expressions can easily be reached as a direct consequence of the skew-symmetric structure of $W_{s s}(\omega)$

$$
e^{T} W_{s s} e=0 \text { and } z^{T} W_{s s} z=0 .
$$

Applying the upper bounds given in (15) and (21) into the second and third lines of (31), respectively, and then competing to squares result in

$$
\begin{aligned}
& \rho_{1}\|e\|-\frac{\rho_{1}^{2}}{\epsilon_{1}}\|e\|^{2}=-\left(\frac{\sqrt{\epsilon_{1}}}{2}-\frac{1}{\sqrt{\epsilon_{1}}} \rho_{1}\|e\|\right)^{2}+\frac{\epsilon_{1}}{4} \leq \frac{\epsilon_{1}}{4} \\
& \rho_{2}\|z\|-\frac{\rho_{2}^{2}}{\epsilon_{2}}\|z\|^{2}=-\left(\frac{\sqrt{\epsilon_{2}}}{2}-\frac{1}{\sqrt{\epsilon_{2}}} \rho_{2}\|z\|\right)^{2}+\frac{\epsilon_{2}}{4} \leq \frac{\epsilon_{2}}{4} .
\end{aligned}
$$

Combining the above derivations allow us to upper bound the right-hand side of (31) as

$$
\dot{V} \leq-\min \left\{\lambda_{\min }\left(K_{e}\right), \lambda_{\min }\left(K_{z}\right)\right\}\|x\|^{2}+\epsilon
$$

where $\epsilon$ was previously defined in (27). Using the definition of $x(t)$ and the upper bound of $V(t)$ given in $(29)$, the upper bound of $\dot{V}(t)$ given (35) can be reformulated as

$$
\dot{V} \leq-\beta V+\epsilon
$$

where $\beta$ was previously defined in (26). The solution of the above differential inequality yields

$$
V(t) \leq V(0) \exp (-\beta t)+\frac{\epsilon}{\beta}(1-\exp (-\beta t))
$$

and from direct application of (29), we can obtain the following upper bound for $x(t)$

$$
\|x(t)\| \leq \sqrt{\frac{a}{b}\|x(0)\|^{2} \exp (-\beta t)+\frac{2 \epsilon}{b \beta}(1-\exp (-\beta t))}
$$


where $a$ and $b$ were previously defined in (24) and (25), respectively. Based on (38) and the definition of $x(t)$, it can be shown that the voltage error term $e(t)$ is bounded as stated in (23). Moreover, applying standard signal chasing argument, we can show that all signals in the closed-loop error system are bounded.

\section{SIMULATION RESULTS}

In order to illustrate the performance of the purposed controller scheme we have performed simulations using MATLAB Simulink simulation tool. DC link voltage is taken as $620 \mathrm{~V}$ and DC link capacitor is assumed to be large enough to keep the DC link voltage constant. Parameters of the low-pass LC filters are taken from $\mathrm{Mu}$ et al. [2011] as

$$
L_{f}=15 \mathrm{mH}, C_{f}=22 \mu \mathrm{F}, r_{f}=0.2 \Omega, R_{L}=20 \Omega
$$

where $R_{L}$ is a resistive load connected to the output of the filter. The amplitude of the reference phase to phase voltage is set to $380 \mathrm{~V}$ and the frequency is $50 \mathrm{~Hz}$. In order to avoid high jumps in the control signal at the beginning, reference signal is designed to be have a soft start. Controller gain matrices are selected as

$$
\begin{array}{cc}
K_{e}=\operatorname{diag}\{2.5,1.93\} & K_{z}=\operatorname{diag}\{26,3.14\} \\
\rho_{1}=\operatorname{diag}\{0.5,0.2\} & \rho_{2}=\operatorname{diag}\{2,2\} \\
\epsilon_{1}=0.1 & \epsilon_{2}=1 .
\end{array}
$$

Simulation results are shown in Figures 2-5. Fig. 2 shows the error signals in d-q synchronous reference frame while three phase output voltage errors are represented in Fig. 3. Fig. 4 presents the input voltage of the output LC filter. Finally, output voltage wave forms of the inverter with output LC filter is presented in Fig. 5.
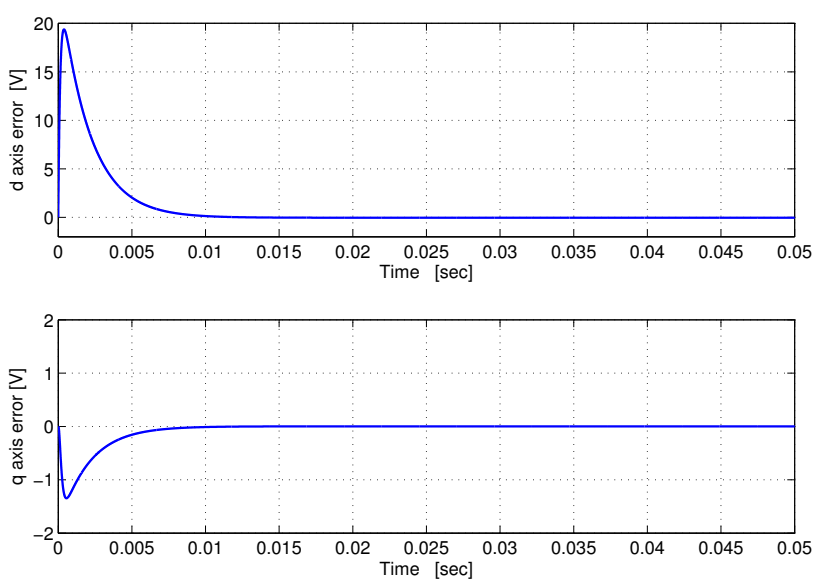

Fig. 2. Error signals in d-q reference frame

\section{CONCLUSION}

In this study, we present a new full state feedback nonlinear robust controller for inverters with output LC filter with uncertain system parameters. Despite the lack of exact knowledge of the filter parameters, proposed controller ensures the desired output wave form with the desired amplitude and frequency for the stand alone and grid connected applications. Stability of the closed-loop

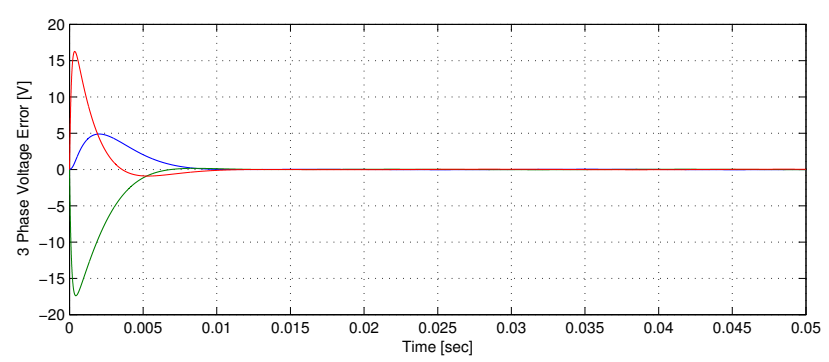

Fig. 3. Error signals in 3 Phase
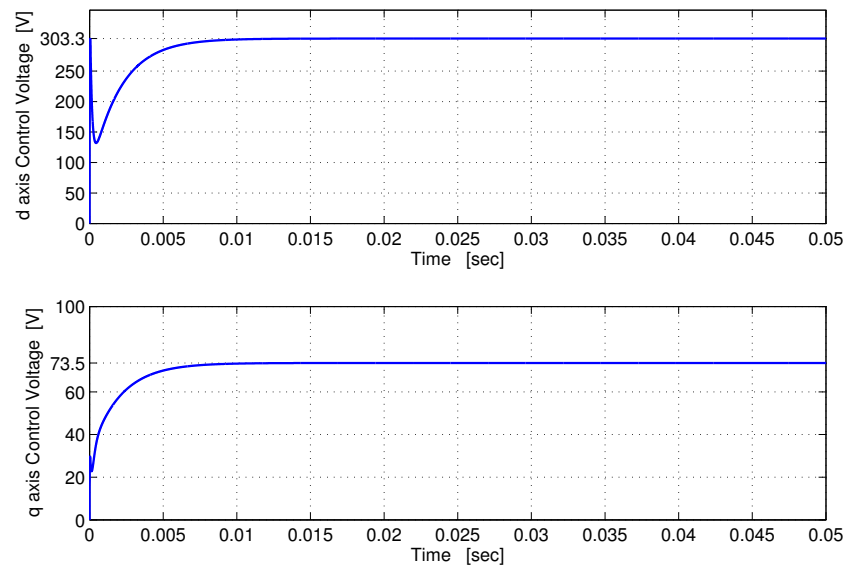

Fig. 4. Control signals in d-q reference frame

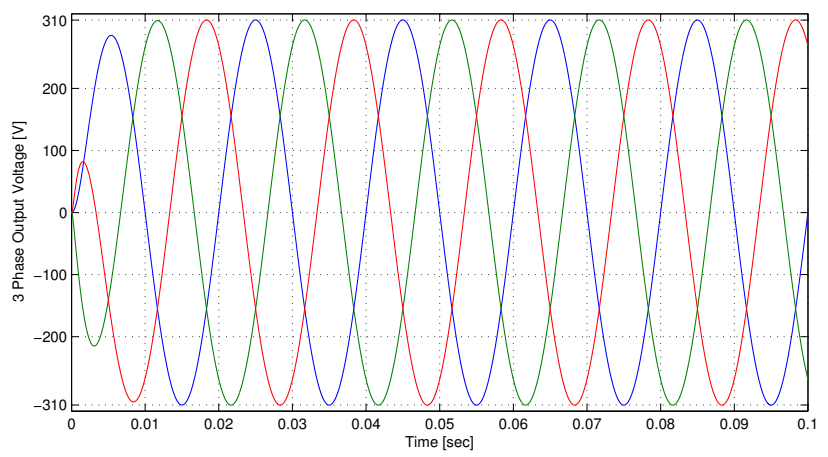

Fig. 5. The output voltage waveform of the inverter

system and boundedness of the signals in the closedloop system is proven via Lyapunov based tools with the help of backstepping procedure. In order to illustrate the performance of the proposed controller, simulation studies have been performed and results of the simulations have been presented.

\section{REFERENCES}

K.H. Ahmed, S.J. Finney, and B.W. Williams. Passive filter design for three-phase inverter interfacing in distributed generation. In Compatibility in Power Electronics, 200\%. CPE '0\%, pages 1-9, 2007.

F. Blaabjerg, R. Teodorescu, M. Liserre, and A.V. Timbus. Overview of control and grid synchronization for distributed power generation systems. Industrial Electronics, IEEE Transactions on, 53(5):1398-1409, 2006. 
P. Cortes, G. Ortiz, J.I. Yuz, J. Rodriguez, S. Vazquez, and L.G. Franquelo. Model predictive control of an inverter with output lc filter for ups applications. IEEE Transactions on Industrial Electronics, 56(6):1875-1883, 2009.

Mohamed a. Eltawil and Zhengming Zhao. Grid-connected photovoltaic power systems: Technical and potential problemsA review. Renewable and Sustainable Energy Reviews, 14(1):112-129, 2010.

G. Escobar, P. Mattavelli, A.M. Stankovic, A.A. Valdez, and J. Leyva-Ramos. An adaptive control for ups to compensate unbalance and harmonic distortion using a combined capacitor/load current sensing. Industrial Electronics, IEEE Transactions on, 54(2):839-847, 2007.

A. Houari, H. Renaudineau, J-P Martin, S. Pierfederici, and F. Meibody-Tabar. Flatness-based control of threephase inverter with output lc filter. Industrial Electronics, IEEE Transactions on, 59(7):2890-2897, 2012.

Dong-Eok Kim and Dong-Choon Lee. Feedback linearization control of three-phase ups inverter systems. IEEE Transactions on Industrial Electronics, 57(3):963-968, 2010.

Hyosung Kim and Seung-Ki Sul. A novel filter design for output lc filters of pwm inverters. Journal of Power Electronics, 11(1):74-81, 2011.

A. Kovari, I. Kadar, and S. Halasz. The influence of inverter control algorithm and dc link voltage on the inverter switching loss. In Industrial Technology, 2004. IEEE ICIT '04. 2004 IEEE International Conference on, volume 2, pages 590-595 Vol. 2, 2004.

O. Kukrer. Deadbeat control of a three-phase inverter with an output lc filter. Power Electronics, IEEE Transactions on, 11(1):16-23, 1996.

R. Lawrence and S. Middlekauff. The new guy on the block. Industry Applications Magazine, IEEE, 11(1): 54-59, 2005.

P. Mattavelli. An improved deadbeat control for ups using disturbance observers. Industrial Electronics, IEEE Transactions on, 52(1):206-212, 2005.

Xiaobin $\mathrm{Mu}$, Jiuhe Wang, Hao Xiang, Yuling Ma, and Dongying Yang. Study on a nonlinear control strategy for three-phase voltage sources pwm dc/ac inverter based on pch model. In Electrical Machines and Systems (ICEMS), 2011 International Conference on, pages 1-4, 2011.

M. Prodanovic and T.C. Green. Control of power quality in inverter-based distributed generation. In IECON 02 [Industrial Electronics Society, IEEE 2002 28th Annual Conference of the], volume 2, pages 1185-1189 vol.2, 2002.

Rong-Jong Wai, Chih-Ying Lin, Wen-Chuan Wu, and Hsin-Ning Huang. Design of backstepping control for high-performance inverter with stand-alone and gridconnected power-supply modes. Power Electronics, IET, 6(4):752-762, 2013. 